\title{
An experimental study of thoriated tungsten cathodes operating at different current intensities in an atmospheric-pressure plasma torch.
}

\author{
J A Sillero, D Ortega, E Muñoz-Serrano and E Casado \\ Departamento de Física, Universidad de Córdoba, 14071 Córdoba, España \\ E-mail: f92simaj@uco.es
}

\begin{abstract}
.
Thoriated tungsten cathodes operating in an open-air plasma torch at current intensities between 30 and $200 \mathrm{~A}$ were experimentally studied. The morphology and composition of the cathode tip after arcing were investigated by scanning electron microscopy and energy-dispersive X-ray spectroscopy. Three relevant zones were found on the cathode tip (spot zone, thorium-depleted zone and thorium-enriched zone), and their dimensions were measured. For each current intensity, the spot temperature $T$ during arcing was measured by one-colour pyrometry, and the spot current density was determined. Exponential growth was seen for the current density as the spot temperature increased. From the experimental data and the energy balance in the presheath, the dependence of the cathode effective work function $\varphi$ on the arc current intensity and its dependence on $T$ were determined. It was found that $\varphi$ increased from $\varphi=2.64 \mathrm{eV}$ when $T \approx 2900 \mathrm{~K}$ to $\varphi=3.06 \mathrm{eV}$ when $T \approx 3700 \mathrm{~K}$. These values are consistent with the presence in the spot of a layer of thorium atoms on the tungsten matrix during arcing. The atomic surface density of the layer determines the value of $\varphi$.
\end{abstract}

\section{Introduction}

The investigation of cathodes is important in order to improve the performance of plasma torches due to the essential role of cathodes as electron emitters and the severe erosion of cathodes caused by the high temperatures attained on the cathode surface. Binary cathodes, formed by a tungsten matrix doped with rare-earth oxides, are of particular interest, as they combine the refractory properties of tungsten with the good electron emission characteristics of the dopant. The processes that take place in such cathodes during arcing are complex due to the interaction between the dopant and the tungsten matrix and between both the dopant and the tungsten with the plasma arc. In spite of the progress achieved in the understanding of binary cathodes, there are still fundamental aspects that are not well understood and require further experimental investigation. For instance, the dependence of the cathode work function on the spot temperature has not yet been satisfactorily established.

Tungsten cathodes doped with thoria are commonly used in industrial applications of plasma torches. Our goal in the present paper is to investigate the behaviour of these cathodes over a wide range of arc current intensities. Thoriated tungsten cathodes have been experimentally studied by different authors [1-11], but in most of the studies, the cathodes were operated with only a few current intensities; therefore, the dependences of the cathode parameters on the arc current intensity $I$ were not found.

In the present paper, we discuss our experimental investigation of thoriated tungsten cathodes operating in the actual working conditions of an industrial plasma torch. We used arc current intensities ranging from $30 \mathrm{~A}$ to $200 \mathrm{~A}$, which allows the investigation of cathode 
behaviour with spot temperatures from about $2900 \mathrm{~K}$ to $3700 \mathrm{~K}$. This covers the operating temperature range of the cathodes.

\section{Experimental setup}

Thoriated tungsten cathodes (tungsten matrix doped with $2 \mathrm{wt} \%$ of $\mathrm{ThO}_{2}$ ) were used in a transferred arc plasma torch (Kemppi model TIG-350 DC-P) operated in open air with a refrigerated cooper anode. The cathodes were cylinders with diameters of $2.4 \mathrm{~mm}$ and cone tips of $68^{\circ}$. The plasma gas was argon with $2 \mathrm{vol} . \%$ of hydrogen. The small amount of hydrogen was added to avoid oxidation of the cathode surface by atmospheric oxygen [9]. The gas flux was $20 \mathrm{slm}$.

A sketch of the experimental setup is shown in figure 1. During arcing, the emission spectrum of the cathode tip surface was registered, and the cathode tip was photographed. A spectrometer with a maximum integration time of $800 \mathrm{~ms}$ and resolution of $0.5 \mathrm{~nm}$ was used. The lens system imaged the cathode surface onto the pinhole without magnification. A standard tungsten ribbon lamp was used to calibrate the spectral response of the system.

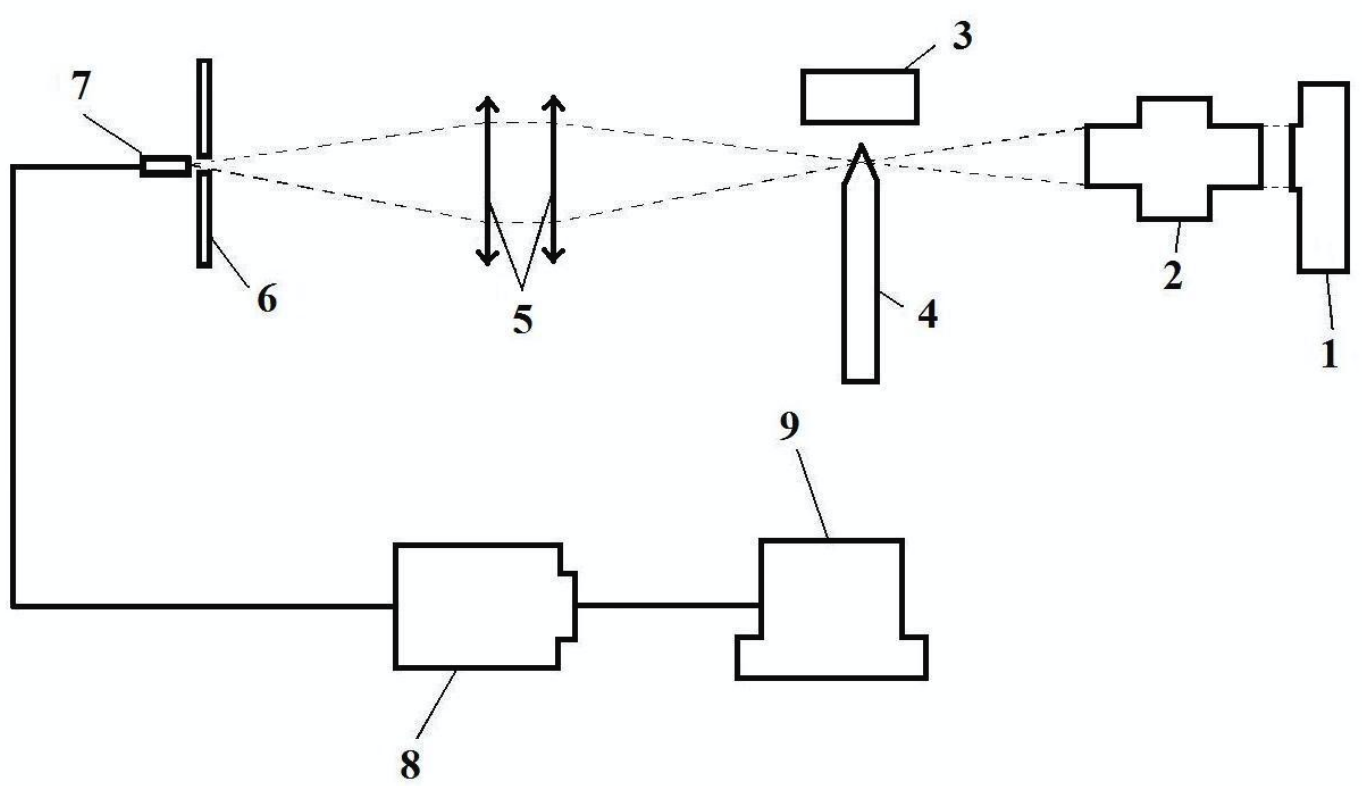

Figure 1. Diagram of the experimental set-up. 1: Digital camera with filters, 2: telemicroscope, 3: anode, 4: cathode, 5: lenses, 6: pinhole, 7: optical fibre, 8: spectrometer, 9: computer.

\section{Experimental procedure}

Thoriated tungsten cathodes operated with arc current intensities of 30, 50, 70, 100, 130, 150 and $200 \mathrm{~A}$ were investigated. With each current intensity, a new cathode was employed for 10 minutes of arcing. The arc voltage was always $22 \mathrm{~V}$, fixed by changing the cathode-anode distance, which varied from $9 \mathrm{~mm}$ at $30 \mathrm{~A}$ to $12 \mathrm{~mm}$ at $200 \mathrm{~A}$. For all current intensities, the arc was stable, and the arc attachment remained immobile at the cathode tip.

The cathode surface temperature was measured by one-colour pyrometry with a wavelength of $721 \mathrm{~nm}$, following the method developed by Zhou et al. [5]. At that wavelength, the error in the temperature measurement due to the continuum radiation of the arc was less than $4 \%$. Moreover, the influence of the line radiation of the elements present in the arc was minimised, as no emission lines of those elements are near $721 \mathrm{~nm}$. The emissivity of the cathode was assumed to be equal to the tungsten emissivity. The temperature dependence of tungsten emissivity at $721 \mathrm{~nm}$ was evaluated from the tables of De Vos [12]. The wavelength calibration of the spectrometer was realised with the $696.5 \mathrm{~nm}$ line of the argon spectrum.

The radiation emitted by a zone of the cathode surface of $10-\mu \mathrm{m}$ diameter and centred at $0.1 \mathrm{~mm}$ of the cathode tip end was collected. As will be shown later, said zone was on the cathode spot. The radiation intensity measurements were performed at 6,8 and 10 minutes after 
the beginning of arcing. Three consecutive measurements were done at each one of those time points. The cathode surface temperature was obtained from the mean of all the measured radiation intensities. The statistical error was $\pm 40 \mathrm{~K}$.

Photographs of the cathode tip were taken at 2-minute periods during arcing. Before and after arcing, the morphology of the cathode surface and its atomic composition were studied by scanning electron microscopy (SEM) and energy-dispersive X-ray spectroscopy (EDX), respectively.

\section{Results and discussion}

4.1 Morphological and atomic composition analyses of the cathode surface

SEM and EDX analyses revealed several zones of different morphologies and atomic compositions on the cathode tip after arcing (figure 2): (I) the spot zone, (II) a thorium-depleted zone, (III) an unaltered zone and (IV) a thorium-enriched zone. Those zones were present after arcing at all examined arc current intensities, although the zone widths depended on the current intensity.

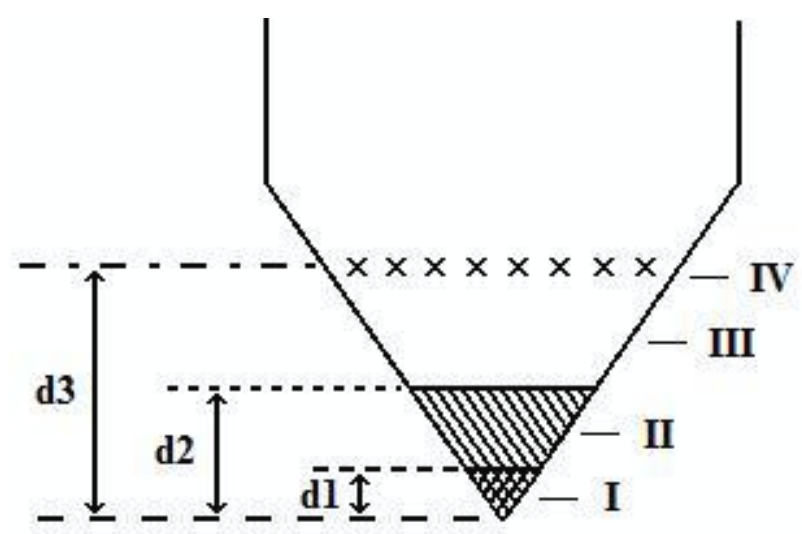

(a)

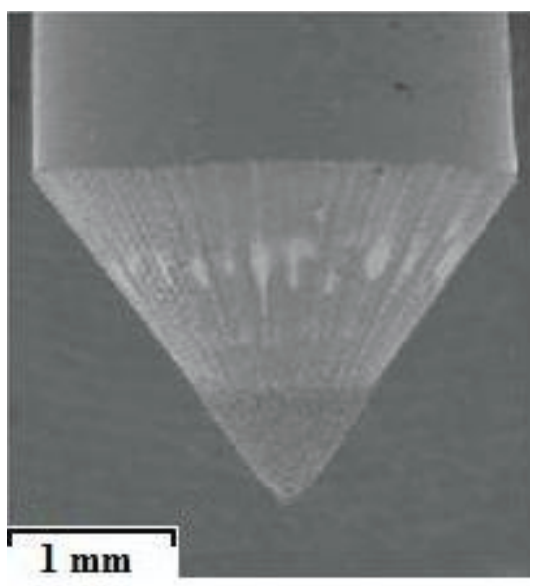

(b)

Figure 2. (a): Zones on the surface of the cathode tip after arcing. Zone I: spot. Zone II: thorium-depleted zone. Zone III: unaltered zone. Zone IV: thorium-enriched zone. (b): SEM micrograph of a cathode after 10 minutes of arcing with 100 A current intensity. Values of $d l$, $d 2$ and $d 3$ are reported in table 1 , and atomic compositions of each zone are shown in table 2.

A micrograph of the spot zone is shown in figure 3a. That zone is characterised by a high porosity due to its high temperature during arcing. It is shown in table 1 that distance $d l$ from the cathode tip end to the end of the spot zone tended to increase as the current intensity increased, although $d l$ values at $100 \mathrm{~A}$ and $130 \mathrm{~A}$ are somewhat high. EDX analyses of the spot zone showed that no thorium was present in this zone after arcing with any current intensity, as can be seen in table 2 .

Table 1. Dimensions of the zones on the cathode tip. $d 1, d 2$ and $d 3$ are the distances from the cathode tip end to the end of the spot, to the end of the thorium-depleted zone, and to the centre of the thorium-enriched zone, respectively. $w$ is the width of the latter zone.

\begin{tabular}{ccccc}
\hline$I(\mathrm{~A})$ & $d I(\mu \mathrm{m})$ & $d 2(\mu \mathrm{m})$ & $d 3(\mu \mathrm{m})$ & $w(\mu \mathrm{m})$ \\
\hline 30 & $105.3 \pm 2.3$ & $214.7 \pm 7.0$ & $408.1 \pm 17.8$ & $114.6 \pm 27.4$ \\
50 & $107.6 \pm 2.8$ & $357.7 \pm 9.8$ & $666.1 \pm 7.5$ & $157.8 \pm 15.5$ \\
70 & $111.5 \pm 3.1$ & $431.7 \pm 8.5$ & $772.9 \pm 10.9$ & $194.4 \pm 15.4$ \\
100 & $126.7 \pm 1.8$ & $560.7 \pm 13.3$ & $1103.9 \pm 25.9$ & $191.7 \pm 17.8$ \\
130 & $118.8 \pm 2.2$ & $697.0 \pm 11.0$ & $1482.7 \pm 29.5$ & $157.2 \pm 44.8$ \\
150 & $117.3 \pm 1.5$ & $742.0 \pm 4.0$ & $1596.9 \pm 12.5$ & $137.3 \pm 18.0$ \\
180 & $127.3 \pm 2.8$ & $821.0 \pm 14.0$ & $1660.1 \pm 10.0$ & $101.9 \pm 14.9$ \\
200 & $130.7 \pm 3.3$ & $882.7 \pm 13.0$ & $1611.3 \pm 10.0$ & $82.0 \pm 25.0$ \\
\hline
\end{tabular}


An SEM micrograph of zone II (thorium-depleted zone) is shown in figure 3b, where tungsten crystals can be seen. EDX analyses showed a low percentage of thorium in zone II, as shown in table 2 . There was practically no presence of thorium in the region of zone II closest to the spot, while in farther regions, some thorium was detected. The values of distances $d l$ and $d 2$, shown in table 1 , demonstrate that zone II width $(d 2-d l)$ increased as the current intensity increased. The obtained results suggest that the depletion of thorium in zone II was mainly caused by thorium evaporation. That evaporation is proportional to the surface temperature, which is high near the spot and increases as the current increases.

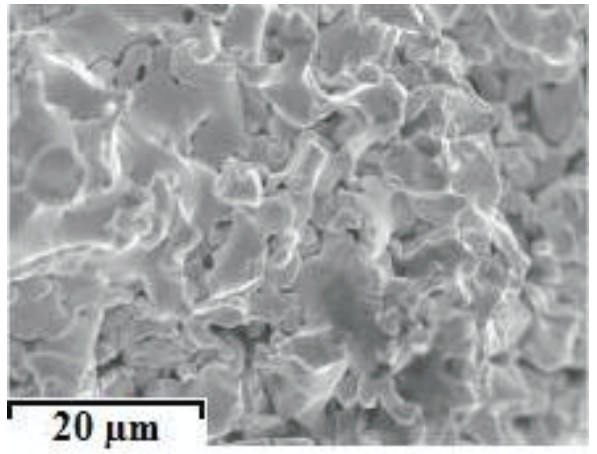

(a)

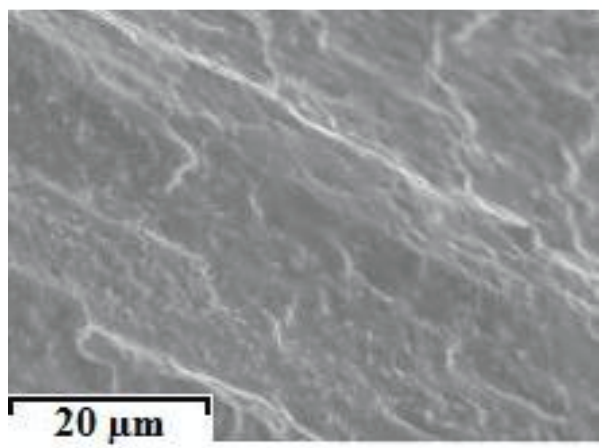

(c)

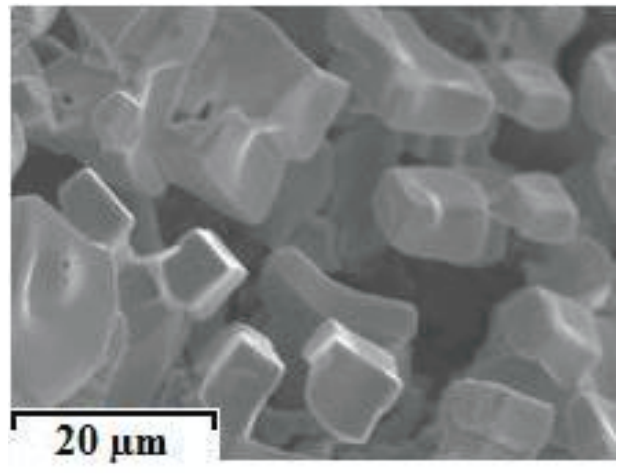

(b)

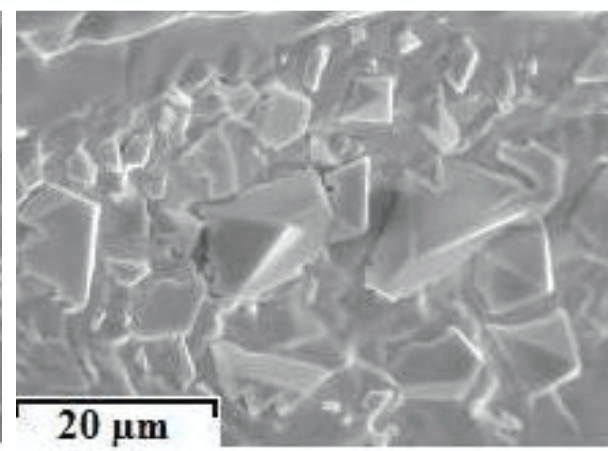

(d)

Figure 3. SEM micrographs of the surface of a cathode after 10 minutes of arcing with a current intensity of $100 \mathrm{~A}$ : (a) the spot, (b) the thorium-depleted zone, (c) the unaltered zone and (d) thorium crystals in the thorium-enriched zone.

Between the thorium-depleted zone and the thorium-enriched zone, a zone with unaltered cathode surface was observed (zone III in figure 2). The morphology of the cathode surface in that zone was similar to the one of a new cathode, as demonstrated by a comparison between figure $3 \mathrm{c}$ and figure 4 . The only difference was that the grooves present on the cathode surface were less sharp in a used cathode than in a new one due to high temperatures attained by cathodes during arcing. The grooves were produced by the whetstone during sharpening of the cathode tip. The atomic composition of zone III was similar to that of a new cathode, as can be seen from the results of EDX analyses shown in table 2.

Table 2. EDX analysis results of three cathodes after arcing with current intensities of 30, 100 and $180 \mathrm{~A}$, respectively. Figures are weight percents of tungsten (W), thorium (Th) and oxygen (O) in the spot (Zone I), in a region of the thorium-depleted zone near the spot (Zone II-a), in a region of the thorium-depleted zone far from the spot (Zone II-b), in the unaltered zone (Zone III) and in the thorium-enriched zone (Zone IV). The weight percentages before arcing were: $94.2 \pm 0.5 \%$ of W, $3.3 \pm 0.4 \%$ of Th and $2.5 \pm 0.3 \%$ of $\mathrm{O}$. 


\begin{tabular}{ccccc}
\hline \multirow{2}{*}{ Zone } & & \multicolumn{3}{c}{ Weight percent } \\
\cline { 3 - 5 } Zone I & Element & $I=30 \mathrm{~A}$ & $I=100 \mathrm{~A}$ & $I=180 \mathrm{~A}$ \\
& $\mathrm{~W}$ & $98.3 \pm 0.4$ & $98.0 \pm 0.7$ & $98.8 \pm 0.6$ \\
& $\mathrm{Th}$ & 0.0 & 0.0 & 0.0 \\
\multirow{3}{*}{ Zone II-a } & $\mathrm{O}$ & $1.7 \pm 0.4$ & $2.0 \pm 0.7$ & $1.2 \pm 0.6$ \\
& $\mathrm{~W}$ & $97.4 \pm 0.6$ & $99.0 \pm 0.5$ & $99.0 \pm 0.5$ \\
& $\mathrm{Th}$ & $1 \pm 0.4$ & 0.0 & 0.0 \\
\multirow{3}{*}{ Zone II-b } & $\mathrm{O}$ & $1.6 \pm 0.4$ & $1.0 \pm 0.5$ & $1.0 \pm 0.5$ \\
& $\mathrm{~W}$ & $95.6 \pm 0.5$ & $97.6 \pm 0.6$ & $95.3 \pm 0.8$ \\
& $\mathrm{Th}$ & $2.2 \pm 0.4$ & $1.0 \pm 0.7$ & $2.5 \pm 0.6$ \\
\multirow{3}{*}{ Zone III } & $\mathrm{O}$ & $2.2 \pm 0.3$ & $1.4 \pm 0.5$ & $2.2 \pm 0.5$ \\
& $\mathrm{~W}$ & $93.9 \pm 0.5$ & $93.5 \pm 0.7$ & $95.0 \pm 0.6$ \\
& $\mathrm{Th}$ & $3.4 \pm 0.4$ & $4.5 \pm 0.6$ & $2.7 \pm 0.4$ \\
& $\mathrm{O}$ & $2.7 \pm 0.3$ & $2.0 \pm 0.5$ & $2.3 \pm 0.3$ \\
\hline \multirow{2}{*}{ Zone IV } & $\mathrm{W}$ & $52.4 \pm 0.60$ & $40.7 \pm 0.6$ & $39.2 \pm 0.9$ \\
& $\mathrm{Th}$ & $37.0 \pm 0.6$ & $46.6 \pm 0.8$ & $48.1 \pm 1.0$ \\
& $\mathrm{O}$ & $10.6 \pm 0.4$ & $12.7 \pm 0.5$ & $12.7 \pm 0.7$ \\
\hline
\end{tabular}

An SEM micrograph of zone IV is shown in figure 3d, where thorium crystals on the cathode surface can be observed. The results of the EDX analysis of zone IV are shown in table 2. In figure $2 \mathrm{~b}$, zone IV corresponds to the zone with clear patches on the cathode surface. In the points between patches, EDX analyses gave results similar to those of a new cathode.

The absence of thorium on the end of the cathode tip agrees with results reported in previous papers $[1,5]$, where a thorium ring corresponding with zone IV was also observed. Zhou et al. [5] considered that the thorium ring is originated by deposition of thorium on the cathode surface, while Sadek et al. [1] considered that the ring is a consequence of the reduction of thoria in the cathode. Our observations support the former hypothesis because no alteration of the cathode surface was detected in the space between regions with thorium crystals.

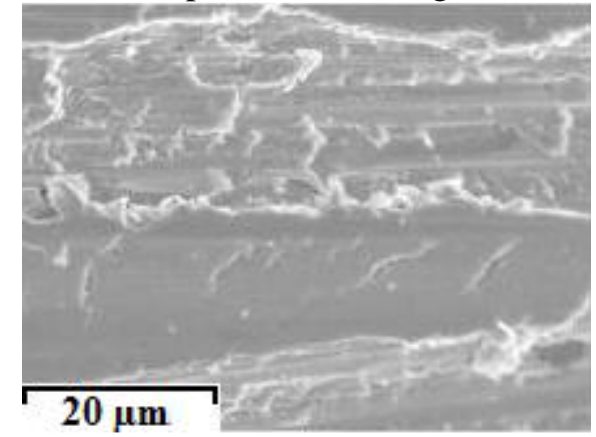

Figure 4. An SEM micrograph of cathode surface before arcing.

\subsection{Spot temperature}

In figure 5, the dependence of the cathode tip temperature on the arc current intensity is shown. The least-squares fitting curve to the experimental points is

$$
T=3712.9-1507 \exp (-I / 52.3),
$$

with $T$ in kelvins and $I$ in amperes. The temperature was measured at a point of the cathode surface located at $0.1 \mathrm{~mm}$ of a new cathode tip end. Therefore, $d l$ values in table 1 indicate that $T$ corresponds to the spot temperature. It is shown in figure 5 that $T$ increased from about 2860 $\mathrm{K}$ at $I=30$ A to $3680 \mathrm{~K}$ at $I=200 \mathrm{~A}$. The steepest increment occurred at low current 
intensities. The $T$ increment guaranteed the larger thermionic emission of electrons required at higher current intensities.

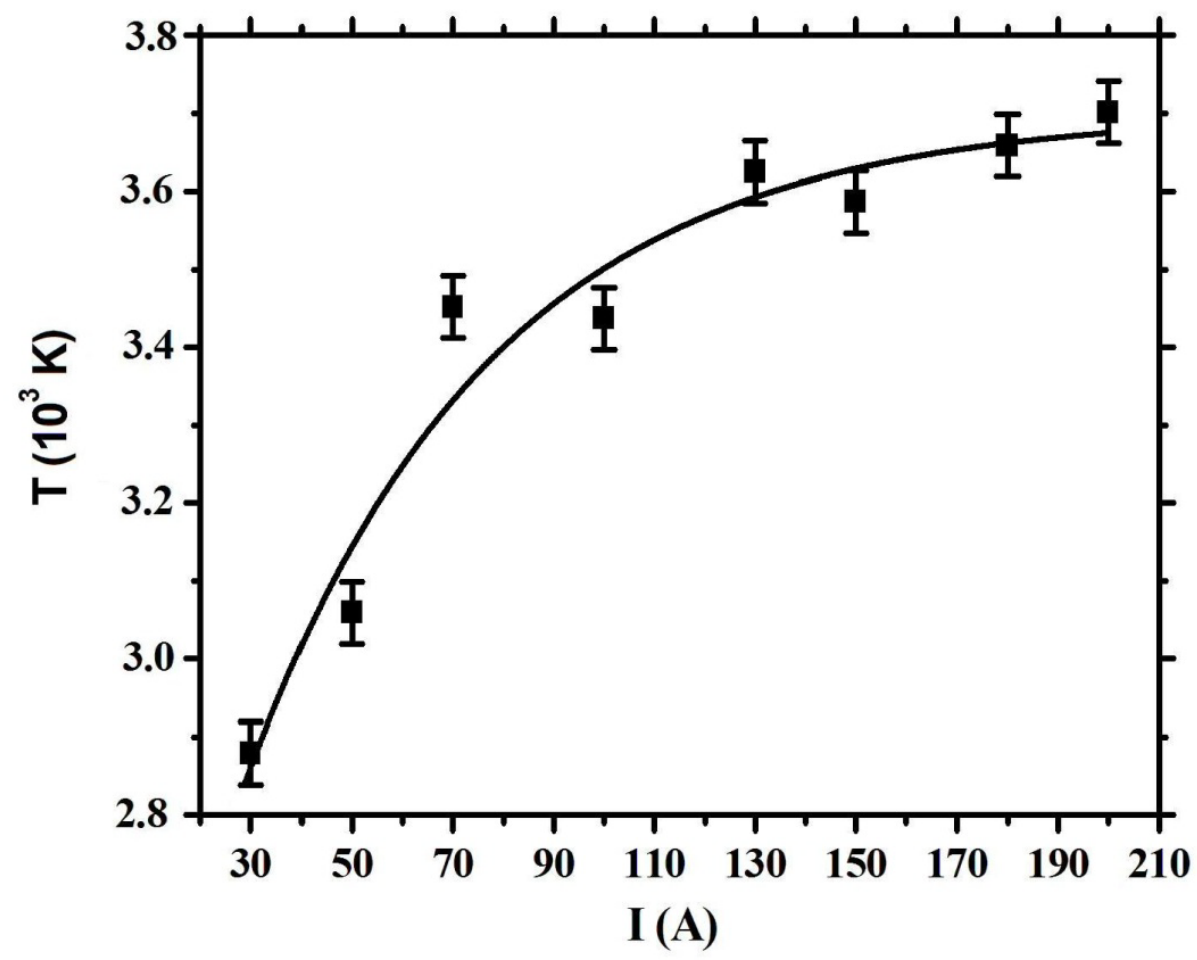

Figure 5. Spot temperature as a function of the arc current intensity.

For $I \geq 80 \mathrm{~A}$, our results are in good agreement with previous measurements of cathode tip temperature $[1,2,4,5-7,11]$. For $I<80$ A, no measurements have previously been reported.

\subsection{Current density}

The spot current density is $j=I / S$, where $I$ is the arc current intensity and $S$ is the spot area. $S$ was evaluated by means of SEM micrographs of the cathode tips after arcing. On each micrograph, a curve $C$ was adjusted to the profile of the spot zone. $C$ contained the point $P$ located at the cathode tip end after arcing. The two branches $C_{I}$ and $C_{2}$ formed by $P$ dividing the curve $C$ were considered, together with the axis $r$ containing $P$ and parallel to the axis of symmetry of a new cathode. The areas $S_{1}$ and $S_{2}$ of the surfaces of revolution generated by rotating $C_{1}$ and $C_{2}$ around $r$ were computed. The spot area $S$ was evaluated as the arithmetic mean of $S_{1}$ and $S_{2}$. The error $\Delta S$ in the evaluation of $S$ was estimated as $\Delta S=\left|S_{2}-S_{1}\right| / 2$. The dependence of $j$ on $I$ is shown in figure 6 . An increase of $j$ is observed as $I$ increases, which is described by the curve $j=1.61 \times 10^{7} I-2.30 \times 10^{4} I^{2}$, with $j$ in A $\mathrm{m}^{-2}$ and with $I$ in A. 


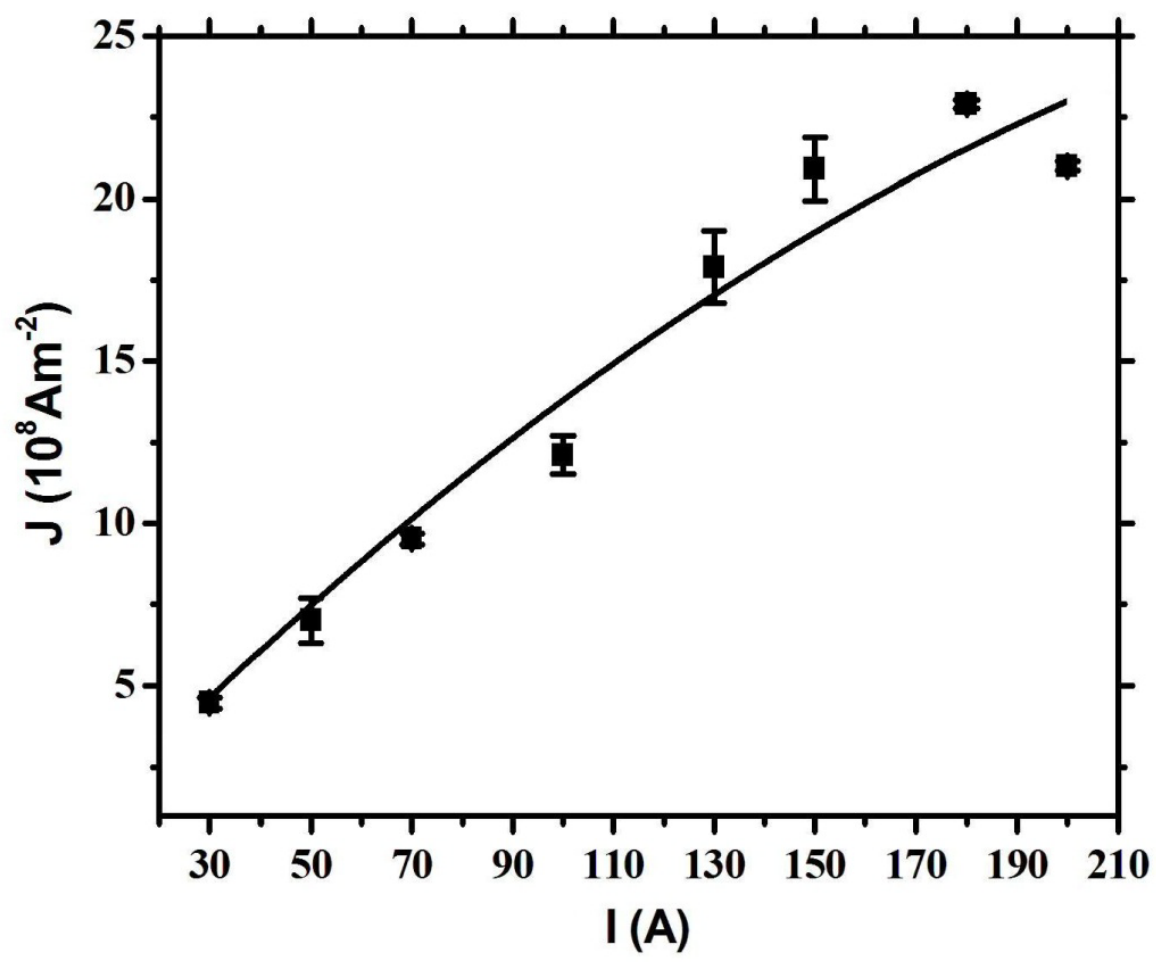

Figure 6. Dependence of the spot current density on the arc current intensity.

From the dependences of $T$ and $j$ on $I$, the dependence of $j$ on $T$ shown in figure 7 was established. In the temperature range investigated, $j$ exhibited an exponential growth described by

$$
j=3.16 \times 10^{8}+4.39 \times 10^{3} \exp (T / 284.5),
$$

with $j$ in $\mathrm{A} \mathrm{m}^{-2}$ and $T$ in $\mathrm{K}$. 


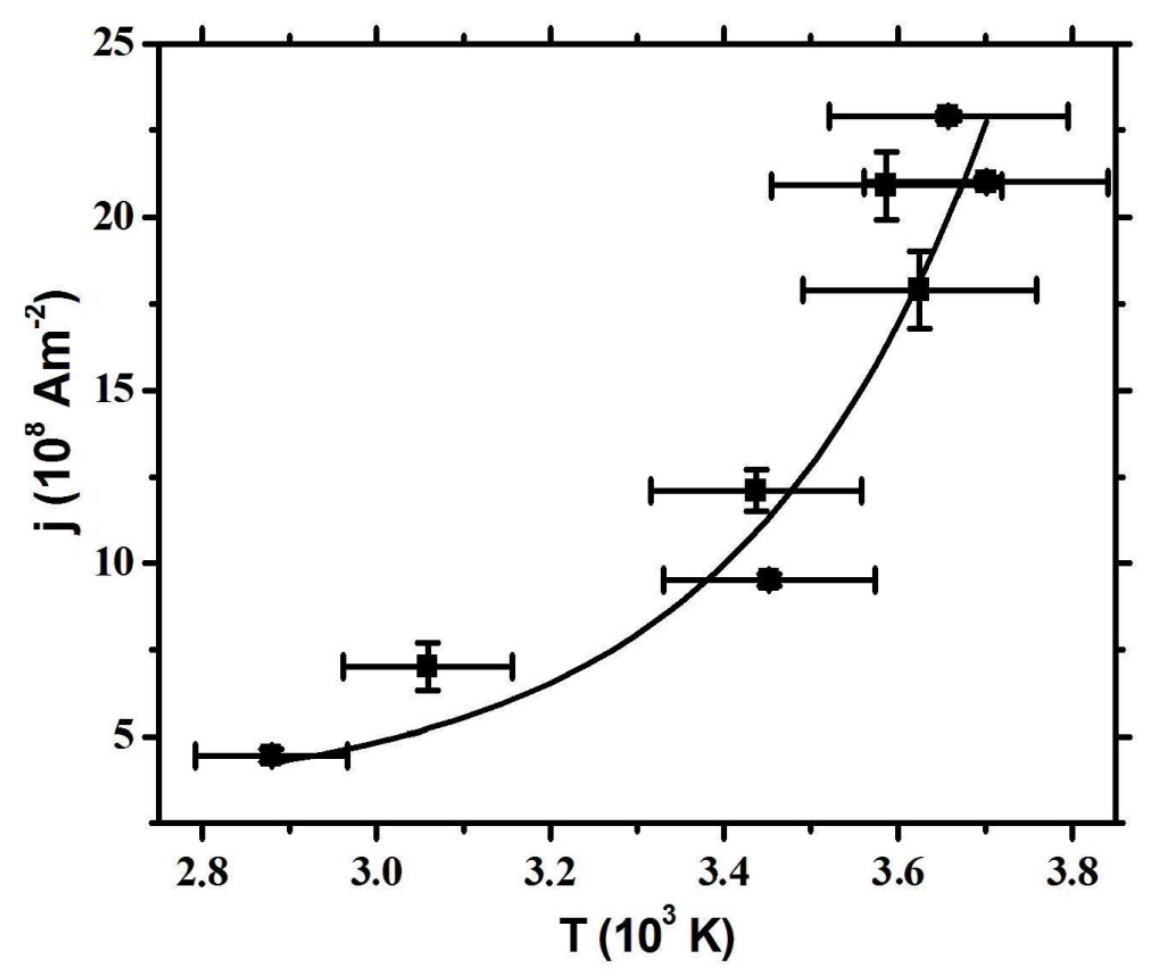

Figure 7. Dependence of the spot current density on the spot temperature.

\subsection{Effective work function}

From the obtained experimental data and the energy balance in the ionization zone of the cathode region, the effective work function of the cathode was determined. For simplicity, multiple-charged ions were not taken into account, and back-diffusion electrons were disregarded because single ionized ions are the main ionic species in the plasma gas [13] and back-diffusion electrons provide a negligible contribution to the current density $[14,15]$. Thus, the energy balance in the ionization zone reads [16]

$$
j_{\mathrm{e}}\left(e U_{\mathrm{c}}+2 k T-3.2 k T_{\mathrm{e}}\right)=j_{\mathrm{i}}\left(X+3.2 k T_{\mathrm{e}}-0.5 e U_{\mathrm{i}}\right),
$$

where $j_{\mathrm{e}}$ is the electron emission current density, $j_{\mathrm{i}}$ is the ion current density, $k$ is the Boltzmann constant, $e$ is the elementary charge, $T_{\mathrm{e}}$ is the electron temperature, $T$ is the spot temperature, $X$ is the ionization energy of the plasma gas atoms, $U_{\mathrm{i}}$ is the potential drop in the ionization zone and $U_{c}$ is the potential drop in the cathode region.

$j_{\mathrm{e}}$ is given by the Richardson-Dushman expression

$$
j_{\mathrm{e}}=A_{\mathrm{D}} T^{2} \exp [-\varphi / k T]
$$

where $A_{\mathrm{D}}=1.2 \times 10^{6} \mathrm{~A} \mathrm{~m}^{-2} \mathrm{~K}^{-2}$ is the Richardson-Dushman constant and $\varphi$ is the effective work function of the cathode. $U_{\mathrm{i}}$ is related to the electron temperature by

$$
U_{\mathrm{i}}=T_{\mathrm{e}} / 7993
$$

( $U_{\mathrm{i}}$ in volts and $T_{\mathrm{e}}$ in kelvins) [17]. As back-scattering electrons has been disregarded,

$$
j_{\mathrm{i}}=j-j_{\mathrm{e}} \text {. }
$$

Substitution of (4), (5) and (6) into (3) allows the evaluation of $\varphi$ as a function of $j$ and $T$, with $T_{\mathrm{e}}$ and $U_{\mathrm{c}}$ as parameters. We used in (3) the values $X=15.76 \mathrm{eV}$ (argon ionization potential), $T_{\mathrm{e}}=20000 \mathrm{~K}$ and $U_{\mathrm{c}}=22 \mathrm{~V}$. The electron temperature in plasmas generated by the plasma torch we used takes values between $18000 \mathrm{~K}$ and $23000 \mathrm{~K}$ [7, 18]. The difference between the actual value of $T_{\mathrm{e}}$ in the plasma and the assumed value $T_{\mathrm{e}}=20000 \mathrm{~K}$ causes a 
variation in $\varphi$ obtained as solution of (3) of less than $1 \%$. As the main contribution to the potential drop in the arc takes place in the cathode region, we set $U_{\mathrm{c}}=22 \mathrm{~V}$. This value for the arc potential drop was fixed for all of the arc current intensities investigated. The exact value of $U_{\mathrm{c}}$ has little influence on the determination of the effective work function. With $U_{\mathrm{c}}$ between 15 and $22 \mathrm{~V}$, the difference between the $\varphi$ values obtained from (3) is less than $2.2 \%$.

The dependence of $\varphi$ on current intensity is shown in figure 8 . The $\varphi$ values were obtained by solving (3) after substitution into that equation of the experimental values of $j$ and $T$ corresponding to each value of $I . \varphi$ changed from $2.63 \mathrm{eV}$ at $30 \mathrm{~A}$ to about $3.02 \mathrm{eV}$ at $200 \mathrm{~A}$. At low current intensities, the effective work function grew sharply, but $\varphi$ increased more slowly as $I$ increased further. $\varphi$ remained almost constant for $I>150 \mathrm{~A}$.

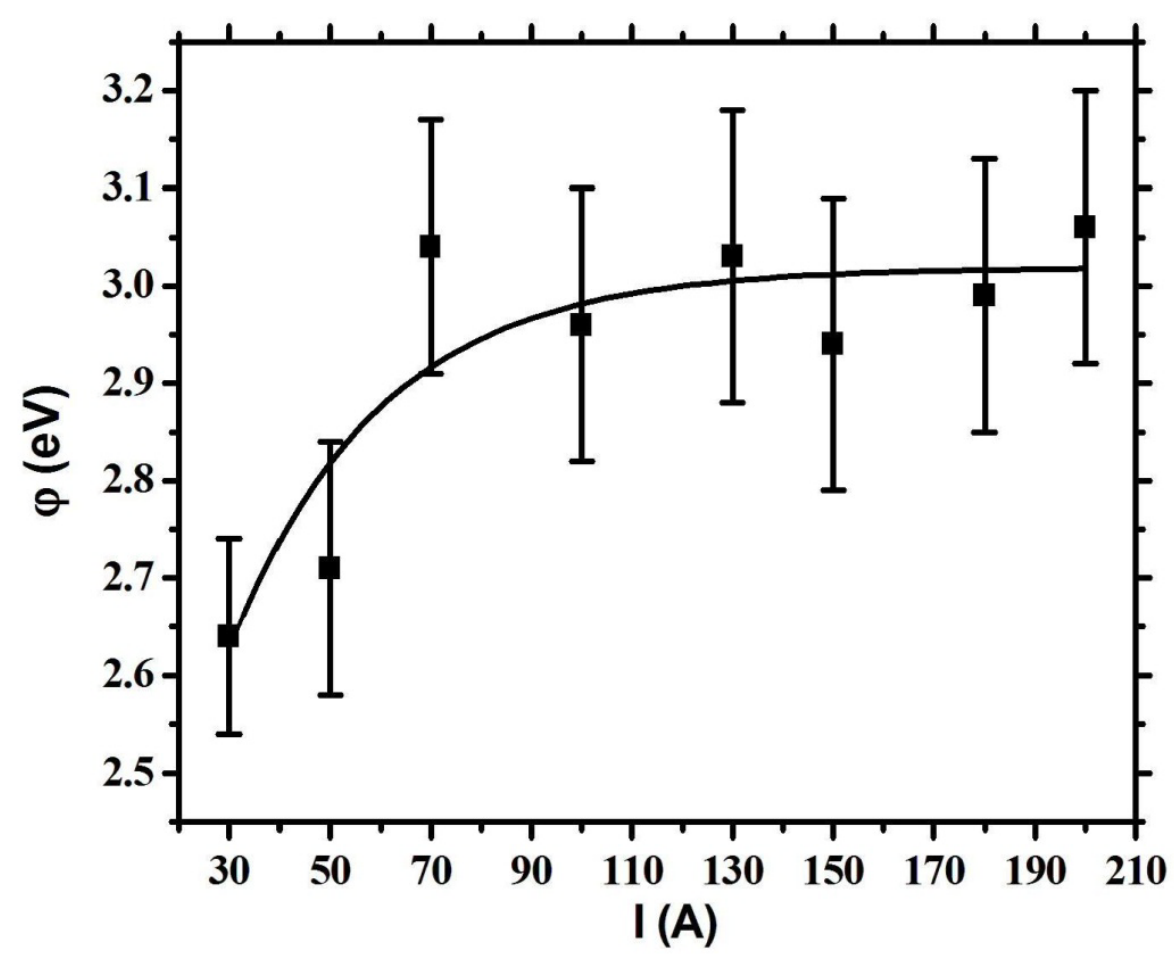

Figure 8. Dependence of the effective work function $\varphi$ on the current intensity $I$. The fitting curve is $\varphi=3.02-1.08 \exp (-I / 29.73)$.

Sadek et al. [1] evaluated the effective work function of thoriated tungsten cathodes with a tip angle of $45^{\circ}$ and found values of $\varphi$ between $2.1 \mathrm{eV}$ and $2.6 \mathrm{eV}$ for current intensities between $100 \mathrm{~A}$ and $200 \mathrm{~A}$. In obtaining those values, the ion contribution to the current density was neglected, which could explain the very low values of $\varphi$ obtained. The values $\varphi=2.6 \mathrm{eV}$ when $I=100 \mathrm{~A}$ and $\varphi=2.8 \mathrm{eV}$ when $I=200 \mathrm{~A}$ were reported by Tanaka et al. [11]. These values were obtained by means of the photoelectric effect and are lower than the ones we obtained for the same current intensities.

The dependence of $\varphi$ on the spot temperature is shown in figure 9. This dependence was obtained from the values of $\varphi$ and $T$ that correspond to each current intensity. $\varphi$ increased from $2.64 \mathrm{eV}$ at $T \approx 2900 \mathrm{~K}$ to $3.06 \mathrm{eV}$ at $T \approx 3700 \mathrm{~K}$. The cause of this behaviour is discussed in the next section. 


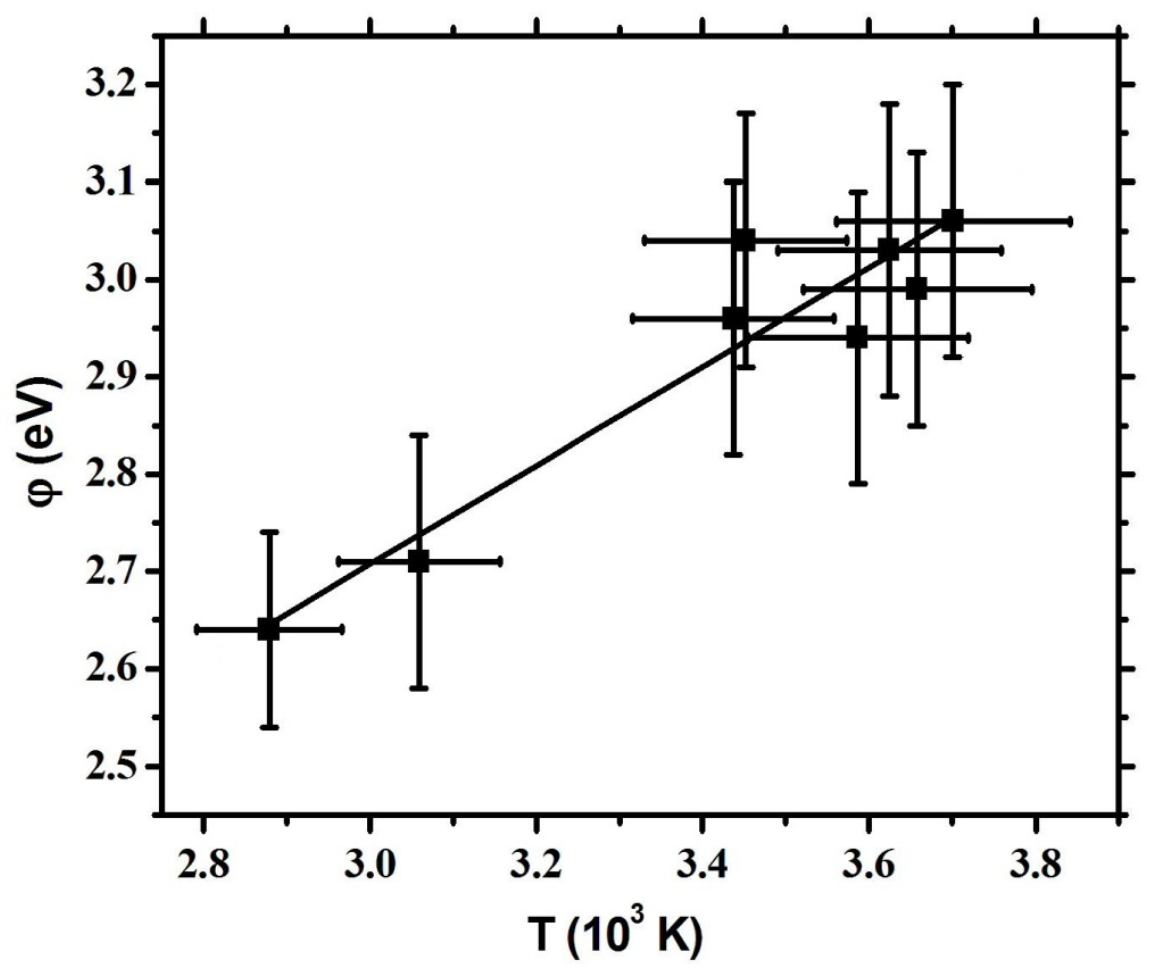

Figure 9. Dependence of the effective work function $\varphi$ on the spot temperature $T$. The fitting curve is $\varphi=1.2+5.0 \times 10^{-4} \mathrm{~T}$.

\subsection{Schottky correction and work function in the absence of an electric field}

The electric field $E$ on the cathode surface reduces the work function $\varphi_{0}$ of the cathode material in the absence of $E$ to the effective work function:

$$
\varphi=\varphi_{0}-\varphi_{\mathrm{S}},
$$

where

$$
\varphi_{\mathrm{S}}=e\left(\frac{e E}{4 \pi \varepsilon_{0}}\right)^{1 / 2}
$$

is the Schottky correction and $\varepsilon_{0}$ is the permittivity of vacuum. From the modelling of the cathode region, $E$ can be evaluated as [16]

$$
E=\sqrt{\frac{2 n_{\mathrm{is}}}{\varepsilon_{0}}}\left\{m_{\mathrm{i}}\left[\frac{v_{+}^{3}-v_{-}^{3}}{6 u_{\mathrm{i}}}-v_{\mathrm{s}}^{2}-\frac{u_{\mathrm{i}}}{3}\right]-k T_{\mathrm{e}}\left[1-\exp \left(\frac{e U_{\mathrm{d}}}{k T_{\mathrm{e}}}\right)\right]\right\}^{1 / 2},
$$

where $m_{\mathrm{i}}$ is the mass of the plasma gas ions, $u_{\mathrm{i}}=\sqrt{k T / m_{\mathrm{i}}}, v_{\mathrm{s}}=\sqrt{k\left(T+T_{\mathrm{e}}\right) / m_{\mathrm{i}}}$,

$v_{ \pm}=\left[\left(v_{\mathrm{s}} \pm u_{\mathrm{i}}\right)^{2}-2 e U_{\mathrm{d}} / m_{\mathrm{i}}\right]^{1 / 2}, U_{\mathrm{d}}=U_{\mathrm{c}}-U_{\mathrm{i}}$ and $n_{\mathrm{is}}$ is the ion density in the sheath-presheath boundary. As $n_{\mathrm{is}}$ is equal to the electron density in that boundary,

$$
n_{\mathrm{is}}=n_{\mathrm{i} \infty} \exp \left(-\frac{e U_{\mathrm{i}}}{k T_{\mathrm{e}}}\right),
$$

where $n_{\mathrm{i} \infty}$ is the ion density in the plasma. At the electron temperature of the plasmas we investigate, the neutral atom density can be neglected in comparison with charged particle density [13]. Therefore, 


$$
n_{\mathrm{i} \infty}=\frac{p}{k\left(T+T_{\mathrm{e}}\right)} .
$$

From equation (9), taking into account (5), (10) and (11), $E$ can be derived for the experimental values of $T$ that correspond to each current intensity. Finally, substitution of $E$ into (8) gives the Schottky correction. With $m_{\mathrm{i}}$ equal to the argon atomic mass, $p=1 \mathrm{~atm}$, $T_{\mathrm{e}}=20000 \mathrm{~K}$ and $U_{\mathrm{c}}=22 \mathrm{~V}$, the value $\varphi_{\mathrm{S}}=0.41 \mathrm{eV}$ was obtained for all of the current intensities investigated. Experimental errors in $T$ introduced in the evaluation of $\varphi_{\mathrm{S}}$ an error of less than $0.3 \%$.

Adding $\varphi_{\mathrm{S}}=0.41 \mathrm{eV}$ to the previously obtained effective work function values (figure 9 ), it was determined that the work function of thoriated tungsten cathodes changed from $\varphi_{0}=3.05 \mathrm{eV}$ at low temperatures $(T \approx 2900 \mathrm{~K})$ to $\varphi_{0}=3.46 \mathrm{eV}$ at high temperatures ( $T \approx 3700 \mathrm{~K}$ ). The work function of thorium deposited on polycrystalline tungsten changes from $3.07 \mathrm{eV}$ when the atomic surface density of thorium is $\sigma_{\max }=4.2 \times 10^{18} \mathrm{~m}^{-2}$ to $4.54 \mathrm{eV}$ (tungsten work function) when there is no thorium on the tungsten surface [19]. Therefore, our results are consistent with the presence of a layer of thorium atoms on the tungsten substrate of the spot during arcing. The dependence of the work function on the surface density of the thorium layer [19] and the obtained $\varphi_{0}$ values indicate that the atomic surface density of thorium on the spot changed from approximately $\sigma_{\max }$ to $0.4 \sigma_{\max }$ as the spot temperature increased from $2900 \mathrm{~K}$ to $3700 \mathrm{~K}$. These atomic surface densities correspond to thorium coverage between 0.5 and 0.2 monolayers, respectively. Most of the neutral atoms evaporated from the cathode are ionized near the spot surface [20]. The balance between re-deposition of those ions on the spot, thorium evaporation and thorium diffusion to the spot from colder regions of the cathode determines the amount of thorium present on the spot during arcing and, therefore, the value of the work function.

\section{Conclusions}

Thoriated tungsten cathodes ( $2 \mathrm{wt} \%$ of thoria) operated with current intensities of 30, $50,70,100,130,150,180$ and 200 A have been experimentally investigated. SEM and EDX explorations of the cathode tip surface showed the presence of three relevant zones: (I) the spot zone (a very porous and thorium-free zone), (II) a thorium-depleted zone close to the spot, and (IV) a thorium-enriched zone formed by thorium crystals deposited on the cathode surface at a certain distance from the thorium-depleted zone. The rest of the cathode surface, which includes the zone between the thorium-depleted and the thorium-enriched zones, is similar to the surface of the cathode before arcing. The dimensions of all the zones were measured.

The dependence of the spot temperature $T$ on the arc current intensity $I$ was found. $T$ increased from $2860 \mathrm{~K}$ when $I=30 \mathrm{~A}$ to $3680 \mathrm{~K}$ when $I=200 \mathrm{~A}$. The increment in $T$ was sharper for $I<130 \mathrm{~A}$. The spot current density $j$ was determined as a function of $I$, and the dependence of $j$ on $T$ was also found. The spot current density exponentially grew from $j=4 \times 10^{8} \mathrm{~A} \mathrm{~m}^{-2}$ to $j=2 \times 10^{9} \mathrm{~A} \mathrm{~m}^{-2}$ as $T$ increased from $2900 \mathrm{~K}$ to $3700 \mathrm{~K}$.

The dependence of the effective work function $\varphi$ on $I$ and the dependence of $\varphi$ on $T$ were obtained from the experimental measurements with the use of the energy balance in the presheath. It was found that $\varphi$ sharply increased from $2.63 \mathrm{eV}$ to $3.01 \mathrm{eV}$ as $I$ increased from 30 A to 150 A. For $I>150$ A, $\varphi$ increased very slowly, reaching a value of $3.02 \mathrm{eV}$ when $I=200$ A. As function of the spot temperature, $\varphi$ increased from $2.64 \mathrm{eV}$ when $T=2900 \mathrm{~K}$ to $3.06 \mathrm{eV}$ when $T=3700 \mathrm{~K}$.

The Schottky correction was evaluated to be $0.41 \mathrm{eV}$ for all the current intensities. Taking said correction into account, the work function $\varphi_{0}$ of the cathode material in the absence of an electric field was found. $\varphi_{0}$ was equal to $3.05 \mathrm{eV}$ when $T=2900 \mathrm{~K}$ and increased to $3.47 \mathrm{eV}$ when $T$ increased to $3700 \mathrm{~K}$. These values of the work function agree with the work function values of a thorium layer on polycrystalline tungsten, the atomic surface density of 
which decreased from about $4.2 \times 10^{18} \mathrm{~m}^{-2}$ to $1.7 \times 10^{18} \mathrm{~m}^{-2}$. Those atomic surface densities correspond approximately to thorium coverage between 0.2 and 0.5 monolayers.

\section{Acknowledgments}

The authors wish to thank Enresa for its financial support of this research.

\section{References}

[1] Sadek A A, Ushio M and Matsuda F 1990 Effect of rare earth metal oxide additions to tungsten electrodes Metall. Trans. A 21A 3221-36

[2] Haidar J and Farmer A J D 1993 A Method for the Measurement of the Cathode Surface Temperature for a High-Current Free-Burning Arc Rev. Sci. Instrum. 64 542-7

[3] Haidar J and Farmer A J D 1994 Large effect of cathode shape on plasma temperature in high-current free-burning arcs J. Phys. D: Appl. Phys. 27 555-60

[4] Haidar J and Farmer A J D 1995 Surface temperature measurements for tungsten-based cathodes of high-current free-burning arcs J. Phys. D: Appl. Phys. 28 2089-94

[5] Zhou X, Ding B and Heberlein J V R 1996 Temperature Measurement and Metallurgical Study of Cathodes in DC Arcs IEEE Trans. Comp. Pack. Manufact. Technol. A $19320-8$

[6] Zhou X and Heberlein J V R 1996 Characterization of the Arc Cathode Attachment by Emission Spectroscopy and Comparison to Theoretical Predictions Plasma Chem. Plasma Process. 16 229S-44S

[7] Zhou X and Heberlein J V R 1998 An Experimental Investigation of Factors Affecting Arc-Cathode Erosion J. Phys. D: Appl. Phys. 31 2577-90

[8] Heberlein J V R 1999 Electrode Phenomena in Plasma Torches Ann. New York Acad. Sci. 891 14-27

[9] Casado E, Colomer V, Sicilia R and Muñoz-Serrano E 2001 An experimental study of the dependence on the current intensity of the erosion of thoriated tungsten cathodes in plasma arcs IEEE Trans. Plasma Sci. 29 888-94

[10] Casado E, Colomer V, Muñoz-Serrano E and Sicilia R 2002 An Experimental Comparison of the Erosion in Tungsten Cathodes doped with different Rare-Earth Elements J. Phys. D: Appl. Phys. 35 992-7.

[11] Tanaka M, Ushio M, Ikeuchi M and Kagebayashi Y 2005 In Situ Measurements of Electrode Work Functions in Free-Burning Arcs During Operation at Atmospheric Pressure J. Phys. D: Appl. Phys. 38 29-35

[12] De Vos J C 1954 A New Determination of the Emissivity of Tungsten Ribbon Physica XX 3 690-714

[13] Pfender E 1978 Electric Arcs and Arc Gas Heaters, in Hirsh M N and Oskam H J (eds) Gaseous Electronics. Volume I: Electrical Discharges, Academic Press, NY

[14] Zhou X and Heberlein J 1994 Analysis of the arc-cathode interaction of free-burning arcs Plasma Sources Sci Technol. 3 564-74

[15] Benilov M S and Cunha M D 2002 Heating of refractory cathodes by high-pressure arc plasmas: I J. Phys. D: Appl. Phys. 35 1736-50

[16] Benilov M S and Marotta A 1995 A model of the cathode region of atmospheric pressure arcs J. Phys. D: Appl. Phys. 28 1869-82

[17] Neumann W 1987 The mechanism of the thermoemitting arc cathode Ergebnisse der Plasmaphysik und der Gaselecktronik Band 8 (Akademic Verlag, Berlin)

[18] Zhu P, Lowke J J and Morrow R 1992 A Unified theory of free burning arcs, cathode sheaths and cathodes J. Phys. D: Appl. Phys. 25 1221-30

[19] Estrup P J, Anderson J and Danforth W E 1966 Leed studies of thorium adsorption on tungsten Surface Science 4 286-98

[20] Ortega D, Sillero Marin J A, Muñoz-Serrano E and Casado E 2009 Simulation of the atomic and ionic densities in the ionization layer of a plasma arc with a binary cathode J. Phys. D: Appl. Phys. 42085202 (8pp) 